\title{
Recurrent Childhood Cerebellar Astrocytoma
}

National Cancer Institute

\section{Source}

National Cancer Institute. Recurrent Childhood Cerebellar Astrocytoma. NCI Thesaurus. Code C8389.

Regrowth of benign and malignant childhood cerebellar astrocytomas that have previously been subjected to surgical resection and less frequently other forms of therapy such as radiation or chemotherapy. Recurrence of benign tumors (e.g., (pilocytic astrocytoma) may respond to a second surgical procedure. 